\title{
The long-term fate of the hip arthrodesis: does it remain a valid procedure for selected cases in the 21 st century?
}

\author{
Matthias U. Schafroth • Reinoud J. Blokzijl • \\ Daniël Haverkamp • Mario Maas • René K. Marti
}

Received: 7 July 2009 /Revised: 4 August 2009 / Accepted: 5 August 2009 /Published online: 21 August 2009

(C) The Author(s) 2009. This article is published with open access at Springerlink.com

\begin{abstract}
Even in current orthopaedic practice, some cases are still not suitable candidates for hip replacement and hip fusion remains the only option in these highly selected patients. In this retrospective study we describe the long-term clinical outcome, quality of life and radiological evaluation of all adjacent joints in a cohort of 47 hip fusions. The main objective of our study was to show the long-term effects of a fusion. Thirty patients were analysed after an average of 18.2 years (range 6.230.5 years) with a mean SMFA of 31.2 (range 9-70). The VAS for pain for the fused hip was an average 1.9 (range $0-8$ ), for the contralateral hip $2.0(0-8)$, for the ipsilateral knee $2.0(0-8)$, for the contralateral knee $1.8(0-8)$ and for the lower back 3.6 (0-8). Average walking distance was 115 minutes (range 10unlimited). Although the hip arthrodesis has lost popularity, it still is an option for the young patient with severe hip disorders, while leaving the possibility to perform a THA at a later stage. If the arthrodesis is performed with an optimal alignment of the leg, complaints from the adjacent joints are minimal, even in the long-term, and an acceptable quality of life can be obtained. We believe that in highly selected cases a hip fusion, even in current practice, is still a valid option.
\end{abstract}

\section{Introduction}

Hip arthrodesis as a solution for hip-related problems is mainly superseded by total hip arthroplasty (THA) and, in

M. U. Schafroth · R. J. Blokzijl • D. Haverkamp $(\bowtie) \cdot$ R. K. Marti Department of Orthopedic Surgery, AMC,

Amsterdam, The Netherlands

e-mail: D.Haverkamp@heupchirurgie.nl

M. Maas

Department of Radiology, AMC,

Amsterdam, The Netherlands the current treatment of hip disorders, there is hardly room left for arthrodesis of the hip. However, we must realise that in certain scenarios in young adults joint preserving therapy is not an option and total hip arthroplasty is a difficult procedure with an increased risk of failure [1-3]. In these selected young patients with severe hip pathology due to trauma or infectious causes an arthrodesis may be a suitable solution with an acceptable outcome [1-4].

There has been considerable literature in the past about the technique and outcome of a hip fusion. Several studies report of pain in adjacent joints in the long term; the most frequent being lower back pain based on degenerative changes reported between $15 \%$ and $100 \%$ of cases [5-9]. Following in percentage are complaints of the ipsilateral knee and less frequent complaints of the contralateral hip and knee [9-11]. The frequency of these complaints seems to be predicted by the alignment of the fusion which should be optimal $[3,6,12]$. In our clinic, we perform hip fusions with our standard technique using a cobra plate; however, in those complex cases where optimal alignment and optimal fixation cannot be achieved by this method we use the technique with a subtrochanteric pendulum osteotomy to achieve the correct alignment. Even in the most difficult cases this method enables the optimal combination between a stable connection of the remaining femoral head-neck and pelvis and an optimal alignment of the leg.

Although a hip fusion is not the most ideal solution it remains an important option for selected scenarios. Apart from the short-term results, attention should be paid to the long-term results in adolescents and young adults. When performing an arthrodesis it should be realised that a future conversion to THA may become desirable. The main objective of our study was to show the long-term effects of fusion on the adjacent joints using our technique. 


\section{Methods and materials}

In the period between 1974 and 1994, 47 hip arthrodesis were performed by the senior author (RKM). The average age at the time of the arthrodesis was 29 years (range 12-55). Nineteen patients were female $(40 \%)$. The hip disorder which led to arthrodesis was septic arthritis in ten patients (including tuberculosis), aseptic necrosis in one, posttraumatic in 21 , congenital luxation in three patients, four patients were secondary to childhood hip disorders and there were other indications in eight.

We used two surgical techniques for performing a hip arthrodesis. We prefer to perform a cobra plate technique, which was initially developed by Schneider and later modified by several others, eliminating the need for the pelvic osteotomy $[13,14]$. The technique we use is similar to this reported technique; however, we pay extra attention to the abductor muscle since it is mandatory to preserve it if a later total hip arthroplasty is a possibility. The abductors are spared by performing a trochanteric osteotomy which is fixed on/over the cobra plate after the arthrodesis is performed. For complex cases in which the optimal combination of fixation of the arthrodesis and perfect limb alignment is not possible, for instance in septic arthritis with severe destruction of the joint or severe osteonecrosis, we performed an arthrodesis with a subtrochanteric pendulum-osteotomy. The arthrodesis is performed in such a position that optimal contact between femoral head and acetabulum is present, regardless of the position of the leg. The arthrodesis is then relieved by a subtrochanteric osteotomy which also allows optimal positioning of the extremity. After-treatment consisted of traction immobilisation for two weeks followed by six weeks of plaster immobilisation. In this study 33 hips were fused with the cobra plate technique and 14 with the arthrodesis with subtrochanteric pendulum-osteotomy technique.

The clinical follow-up consisted of a thorough clinical investigation and the short musculoskeletal function assessment (SMFA) questionnaire [15]. The SMFA is a wellvalidated, universal, self-reported health status questionnaire. The SMFA questionnaire is developed for clinical assessments of the impact of treatment in groups of patients who have musculoskeletal disease or injury. The SMFA questionnaire consists of the dysfunction index, which has 34 items for the assessment of patient function, and a bother index, which has twelve items for the assessment of how much patients are bothered by functional problems.

Since a hip arthrodesis can have a negative influence on the contralateral hip joint, the back and knees, we included these adjacent joints in this analysis. The clinical investigation consisted of: range of motion of hips and knees, mobility of the back, laxity of the knee joint, leg length discrepancies and quadriceps strength. The mobility of the back was measured using a standardised Mildenberg fingerfloor method and the Schöber test. The Schöber test was conducted with the patient standing upright; a mark was made at the height of L5. A second mark was made $10 \mathrm{~cm}$ above and a third mark $5 \mathrm{~cm}$ below the first mark. The patient was asked to bend forward with the knees straight, reaching as far as possible towards his/her toes. The increase between the upper and lower mark was taken as a measurement of lumbar flexion. The finger-floor measurements were taken upon the first attempt of the patient to bend forward with extended knees. Quadriceps strength was graded from 0 to 5 according to the British Medical Research Council scale. Subjective outcome measurement visual analogue scores (VAS) were obtained for pain in hips, knees and back. VAS scores were also obtained for satisfaction with the results of the arthrodesis and for the eagerness to have the arthrodesis converted to THA. Additional questions concerning work, walking distance, problems with sitting and problems with socks and shoes were posed.

For both knee joints, the International Knee Documentation Committee (IKDC) knee examination form was used to evaluate secondary knee problems. This form results in four groups: group $\mathrm{A}$ is normal knees, group $\mathrm{B}$ nearly normal knees, group $\mathrm{C}$ abnormal knees, and group $\mathrm{D}$ severely abnormal knees.

For the radiological analysis, weight bearing X-rays of pelvis, both knees and lumbar spine were used. The radiographs were judged for the presence of nonunions. The contralateral hip and both knees were scrutinised for signs of osteoarthritic changes which were scored according to Kellgren and Lawrence for the medial compartment, the lateral compartment and the patello-femoral compartment [16]. For the knees, the Ahlback score was also used for the medial and lateral compartment separately [17]. On the lumbar spine X-rays the presence of osteophytes as an indicator for osteoarthritic changes were scored; the percentage of joint-space narrowing between the vertebrae and the presence of olisthesis and scoliosis were documented.

\section{Results}

After an average follow up of 18.9 years (range 5.6-31.4), six patients had died. Five patients could not be traced for follow-up. Seven hips were eventually converted to THA of which one was performed to facilitate a total knee arthroplasty on the same side. The remaining 30 patients were invited for follow-up and intensively screened.

In this group of difficult hip disorders seven patients developed a postoperative complication. One patient suffered from a deep venous thrombosis which was treated with anticoagulants. In two patients a deep infection occurred, of which both received the arthrodesis for a 
destructive septic arthritis. In one of them a revision operation was performed with debridement of the infection and surgical drainage; the infection resolved but resulted in a nonunion. The second infection was treated conservatively, and after 24 years of follow-up a fistula and deep infection were still present. Besides the nonunion after infection, in five other patients a nonunion was diagnosed, and in three patients one re-arthrodesis was sufficient to achieve union, while in one patient two re-arthrodesis were performed to achieve union. One patient was left untreated because of co-morbidity for which this arthrodesis was performed for severe post-traumatic destruction; however, the same trauma led to a post-traumatic ankylosis of the ipsilateral knee, a paresis of the contralateral arm and permanent brain damage (mainly cognitive).

Clinical and radiological follow-up was obtained for 30 patients after an average of 18.2 years (range 6.2-30.5) (Fig. 1). Clinically, the SMFA showed a mean total score of 31.2 (range 9.0-70.0), the subscore dysfunction an average of 29.1 (range 8.0-70.5) and the subscore limitations an average of 37.3 (range 9.0-84.0). The clinical and radiological results for the arthrodesis and adjacent joint will be discussed separately.

The clinical and radiological investigation of the fused hip joint showed an average VAS for pain of 1.9 (range 0.0-8.0). Satisfaction was reasonable with an average VAS of 7.0 (range 3.0-10.0). The average alignment of the arthrodesis was $19^{\circ}$ flexion $(5 ; 30),-1^{\circ}$ of abduction $(-10$; $10)$ and $2^{\circ}$ external rotation $(-10 ; 15)$. An average leg length discrepancy of $-3.3 \mathrm{~cm}$ (range -11.5 to 2 ) was present. Walking distance averaged 115 minutes (range 10unlimited) for which one patient needed two crutches and two patients needed one crutch for support. Twenty-three patients experienced difficulties in putting on their shoes, in 17 of them this was caused by the inability to tie their shoelaces. Eight patients experienced difficulties in putting on their socks. On radiological examination six hips showed signs of a nonunion. Of these, three had no complaints of the fused hip (VAS pain 0,0,3), and one patient with a nonunion after infection had a VAS of 6 . The two remaining patients reported VAS of 6 and 7 for pain; however, they performed full time heavy labour (North Sea fisherman and factory worker). The VAS for eagerness to have their fusion converted to a THA was 5.4 average (range 0-10).

The contralateral hip joint on clinical examination showed an average VAS for pain of 2.0 (range 0-8). Eight patients (24\%) had a VAS higher than 3 . One contralateral hip underwent an intertrochanteric shortening osteotomy and one was converted to a THA. The average range of motion was: for flexion $104^{\circ}$ (range $30-140^{\circ}$ ), extension $0.2^{\circ}\left(0-5^{\circ}\right)$, abduction $25^{\circ}\left(10-40^{\circ}\right)$, adduction $17^{\circ}\left(0-30^{\circ}\right)$, external rotation $27^{\circ}\left(5-60^{\circ}\right)$ and internal rotation $16^{\circ}\left(0-30^{\circ}\right)$.
On clinical examination the ipsilateral knee joint showed an average VAS for pain of 2.0 (range 0-8). Seven patients $(21 \%)$ had a VAS higher than 3. Quadriceps strength was optimal (grade 5) in all. Average range of motion for flexion was $125^{\circ}\left(20-160^{\circ}\right)$ and for extension $2^{\circ}\left(0-10^{\circ}\right)$. On radiological examination three knees showed severe osteoarthritic changes with a grade $4 \mathrm{~K}-\mathrm{L}$ and IV Ahlback in both compartments, and in one knee only in the lateral compartment. A grade 3 or $4 \mathrm{~K}-\mathrm{L}$ OA in the patello-femoral compartment was present in four knees. The IKDC knee examination form showed a grade $\mathrm{A}$ in nine knees, grade $\mathrm{B}$ in 14 knees, grade $C$ in four knees and grade $D$ in eight knees.

On clinical examination the contralateral knee joint showed an average VAS for pain of 1.8 (range 0-8). Seven patients $(21 \%)$ had a VAS higher than 3. Quadriceps strength was optimal (grade 5) in all. Average range of motion for flexion was $140^{\circ}\left(100-160^{\circ}\right)$ and for extension $1^{\circ}(-10$ to $20^{\circ}$ ). On radiological examination one knee showed severe osteoarthritic changes with a grade $4 \mathrm{~K}-\mathrm{L}$ and a grade IV Ahlback in both compartments, and in one knee only in the lateral compartment. A grade 3 or $4 \mathrm{~K}-\mathrm{L}$ OA in the patellofemoral compartment was present in three knees. The IKDC knee examination form showed a grade $\mathrm{A}$ in 23 knees, grade $\mathrm{B}$ in five knees, grade $\mathrm{C}$ in five knees and grade $\mathrm{D}$ in three knees. There was a significant differences in the outcome of the IKDC examination form (laxity) for the ipsilateral and contralateral knees (Chi-square $p<0.001)$.

Clinical investigation of the lower back showed an average VAS for pain of $3.6(0-8)$ (Table 1$)$. Fourteen patients $(42 \%)$ had a VAS higher than 3. The average Schöber test was 6.1 (3-10.5), and the standardised Mildenberg finger-floor method averaged $32.5 \mathrm{~cm}$ (15-51). Radiological examination of the sacro-iliacal joint showed three joints with severe arthrosis (one grade 3 and two grade $4 \mathrm{~K}-\mathrm{L}$ ) on the ipsilateral side, and two (both grade $3 \mathrm{~K}-\mathrm{L}$ ) on the contralateral side. The lumbar spine showed signs of $\mathrm{OA}$ in the form of osteophytes in 12 backs. In two spines an olisthesis was present, one grade 1 and one grade 2. Ten AP lumbar spine showed a scoliosis, of which eight had the convexity on the opposite side of the arthrodesis.

\section{Discussion}

Although arthrodesis has been forced into the background by the successful THA, it still remains a valid option for specific difficult problems [3]. This descriptive study of our fusion techniques shows a good outcome measured by the SMFA and a good VAS for pain for all related joints after an average of 19 years of follow-up. Especially lower back pain seems to occur frequently after a hip fusion in the long term, since $42 \%$ of our patients had a VAS for pain of 3 and higher for lower back pain. 
Fig. 1 a A 17-year-old boy with a central hip luxation.

b Postoperative situation after acetabulum reconstruction.

c Postoperative situation after fusion with a cobra plate.

d-h X-rays of pelvis, knees and lumbar spine 28 years postoperatively. The arthrodesis was converted to a total hip replacement (THR) 20 years postoperatively (on the contralateral hip a shortening at intertrochanteric level was performed 1 year after the fusion)
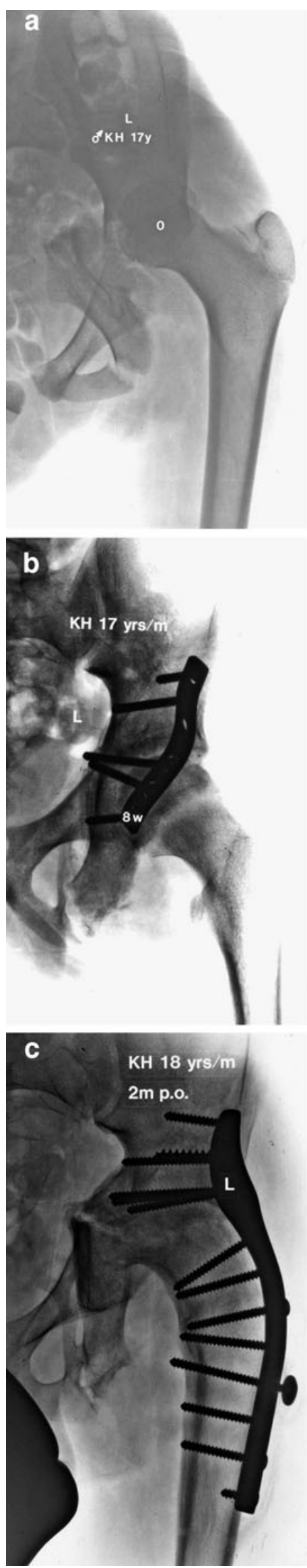
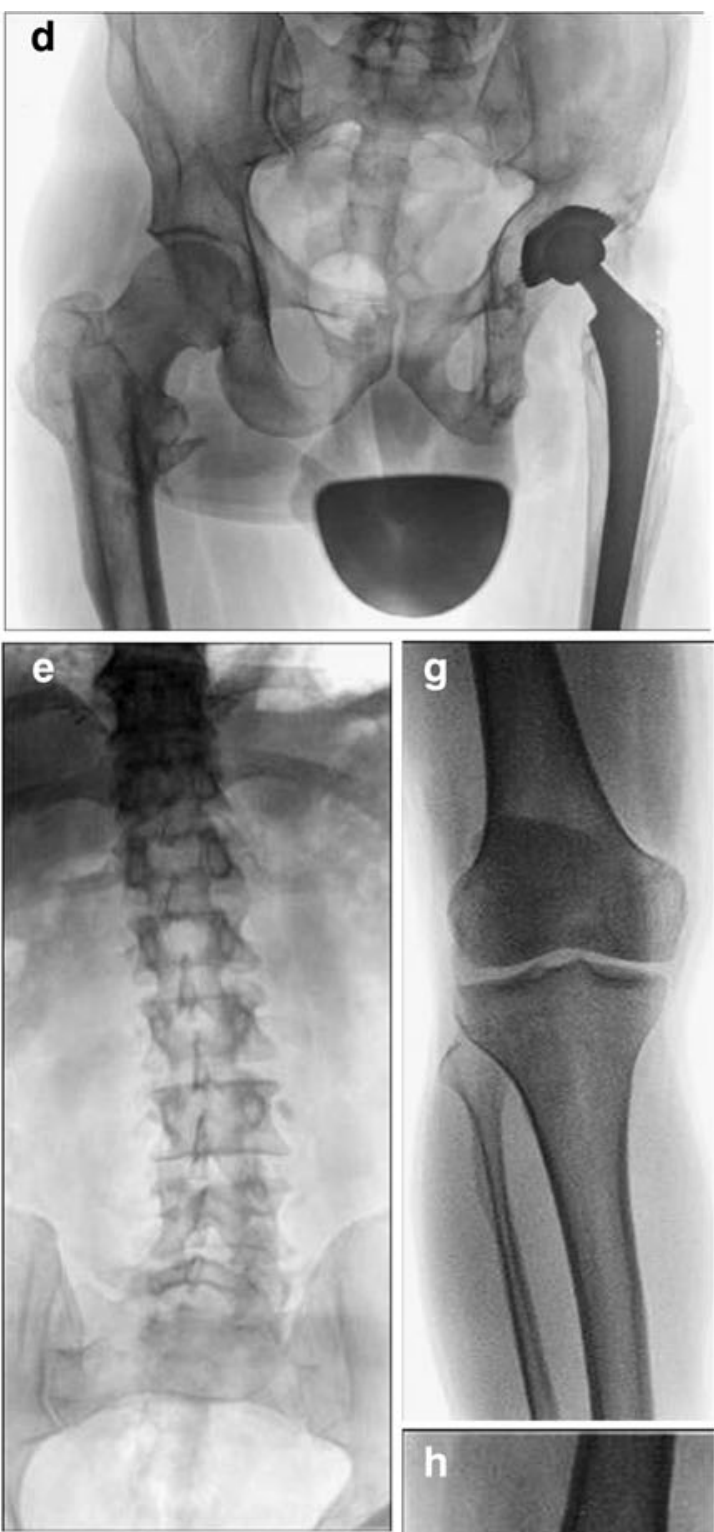

g
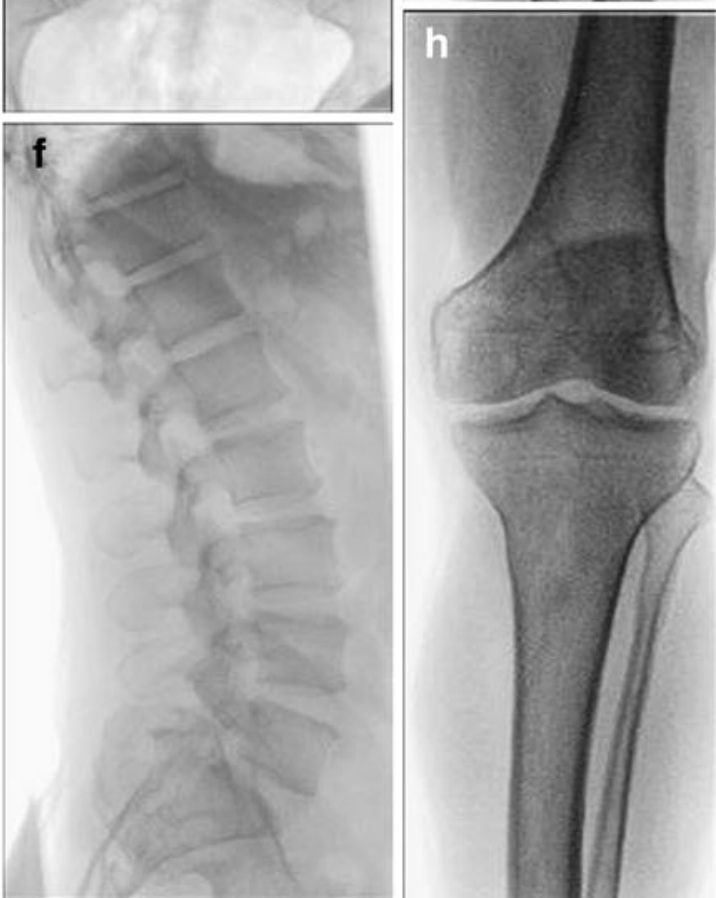
Table 1 Results per operation technique

SFMA short musculoskeletal function assessment, avg average, VAS visual analogue score

\begin{tabular}{lll}
\hline Outcome & $\begin{array}{l}\text { Cobra plate } \\
\text { technique }(n=33)\end{array}$ & $\begin{array}{l}\text { Arthrodesis with subtrochanteric } \\
\text { osteotomy }(n=14)\end{array}$ \\
\hline Complications & $5(15 \%)$ & $2(14 \%)$ \\
Infection & $2(6 \%)$ & 0 \\
Nonunion & $3(9 \%)$ & $2(14 \%)$ \\
SMFA total (avg, range) & $32.4(9-66)$ & $32.2(9-70)$ \\
SMFA dysfunction (avg, range) & $29.8(8-65)$ & $30.9(9-71)$ \\
SMFA bother (avg, range) & $41.1(11-93)$ & $40.0(9-84)$ \\
VAS fused hip (avg, range) & $2.6(0-9)$ & $1.6(0-7)$ \\
Walking distance (avg, range in minutes) & $105(5-$ unlimited) & $90(15-$ unlimited) \\
VAS contralateral hip (avg, range) & $2.4(0-8)$ & $1.7(0-5)$ \\
VAS ipsilateral knee (avg, range) & $2.1(0-8)$ & $1.6(0-8)$ \\
VAS contralateral knee (avg, range) & $1.6(0-8)$ & $1.7(0-8)$ \\
VAS lower back (avg, range) & $4.1(0-9)$ & $1.9(0-7)$ \\
\hline
\end{tabular}

The cobra head plate technique is our first choice since it provides a high fusion rate and allows early mobilisation. For some indications, including severe unilateral destruction of the femoral head in young patients with severe loss of bone stock, which can occur in chronic septic arthritis or osteonecrosis, a cobra head plate arthrodesis does not provide the necessary stability. For these selected patients we performed the arthrodesis technique with a subtrochanteric pendulum osteotomy. This method enables the optimal combination between a stable connection of the remaining femoral head-neck and pelvis and an optimal alignment of the leg even in the most difficult cases.

The fact that an arthrodesis is only indicated in difficult cases is probably the best explanation for the relatively high complication rate in this group of patients. The deep infections only occurred after septic arthritis. A second important complication is the occurrence of nonunions in our patient group. At follow-up, six hips showed signs of a nonunion, although in three of them a real nonunion was unlikely due to the absence of symptoms. Two other patients with signs of a nonunion indicated pain in the fused hip (VAS $6 \& 7$ ), but were able to perform full time heavy labour. Using these two techniques for these selected patients our nonunion rate of $6 \%$ can be considered low $[1,2]$.

It is known that an arthrodesis of any joint has influence on the adjacent joints. These effects can be minimized by performing an optimal alignment of the affected extremity. In an ideal arthrodesis this alignment is adjusted to the patient's preferences. In patients with a mainly sitting occupation more flexion is added than in patients with a standing profession. The adjacent hip joint and both knee joints did not suffer from the arthrodesis in a way that they caused pain or discomfort. However, we noticed that significantly more knees on the ipsilateral side showed an increased laxity (IKDC knee examination form). This coping mechanism of the knee did not cause any clinical complaints. No differences were found in the VAS scores for the contralateral and ipsilateral knees. In 2000, Karol et al. showed in a detailed gait analysis that excessive motion of the lumbar spine and the ipsilateral knee were present in patients with a fused hip [18]. These excessive movements caused complaints in the ipsilateral knee and lower back in their group of seven patients. Our data supports the fact that a fusion causes more lower back complaints in the long term (42\% with a VAS higher than 3$)$; however, we could not confirm the occurrence of more complaints in the ipsilateral knee.

In our study seven arthrodeses (15\%) were eventually converted to a THA (Fig. 1), all with a good result. In the literature several studies can be found regarding conversion of an arthrodesis to a THA, showing identical results to primary THA in patients older than $50[1,19,20]$. Although primarily THA may not be a solution for these patients, an arthrodesis can bridge the gap to a future THA, while generating a good quality of life in the meantime. We asked our patients how eager they were to convert their arthrodesis to a THA and $13(43 \%)$ saw no reasons for conversion at long-term follow-up, indicating that these patients were content with their current quality of life.

We found that the mobility and quality of life were good for patients with an arthrodesis. The average walking time was almost two hours (115 minutes), while ten patients were able to walk for at least three hours, and one patient even participated in marathon running. This indicates that a hip arthrodesis should not necessarily mean a reduced walking ability.

Although hip arthrodesis has lost it popularity it still is an option for young patient with severe hip disorders, while leaving the possibility to perform a THA at a later stage. If the arthrodesis is performed with an optimal alignment of the leg, complaints from the adjacent joints are minimal, even in the long term, and an acceptable quality of life can be obtained. 
Open Access This article is distributed under the terms of the Creative Commons Attribution Noncommercial License which permits any noncommercial use, distribution, and reproduction in any medium, provided the original author(s) and source are credited.

\section{References}

1. Beaule PE, Matta JM, Mast JW (2002) Hip arthrodesis: current indications and techniques. J Am Acad Orthop Surg 10:249-258

2. Duncan CP, Spangehl M, Beauchamp C, McGraw R (1995) Hip arthrodesis: an important option for advanced disease in the young adult. Can J Surg 38(Suppl 1):S39-S45

3. Stover MD, Beaule PE, Matta JM, Mast JW (2004) Hip arthrodesis: a procedure for the new millennium? Clin Orthop Relat Res 418:126-133

4. Courpied JP, Postel M (1983) Indications for arthrodesis of the hip in the adult. Int Orthop 7:159-164

5. Callaghan JJ, Brand RA, Pedersen DR (1985) Hip arthrodesis. A long-term follow-up. J Bone Jnt Surg Am 67:1328-1335

6. Sponseller PD, McBeath AA, Perpich M (1984) Hip arthrodesis in young patients. A long-term follow-up study. J Bone Jnt Surg Am 66:853-859

7. Stewart MJ, Coker TP Jr (1969) Arthrodesis of the hip. A review of 109 patients. Clin Orthop Relat Res 62:136-150

8. Stinchfield FE, Cavallaro WU (1950) Arthrodesis of the hip joint; a follow-up study. J Bone Jnt Surg Am 32A:48-58

9. Sofue M, Kono S, Kawaji W, Homma M (1989) Long term results of arthrodesis for severe osteoarthritis of the hip in young adults. Int Orthop 13:129-133
10. Gore DR, Murray MP, Sepic SB, Gardner GM (1975) Walking patterns of men with unilateral surgical hip fusion. J Bone Jnt Surg Am 57:759-765

11. Hauge MF (1973) The knee in patients with hip joint ankylosis. Clinical survey and bio-mechanical aspects. Acta Orthop Scand 44:485-495

12. Roberts CS, Fetto JF (1990) Functional outcome of hip fusion in the young patient. Follow-up study of 10 patients. J Arthroplasty 5:89-96

13. Schneider R (1974) Hip arthrodesis with the cobra head plate and pelvic osteotomy. Reconstr Surg Traumatol 14:1-37

14. Wagner M, Wagner H (1996) Hip joint arthrodesis using the cobra plate. Indication, technique, outcome. Orthopade 25:129139

15. Swiontkowski MF, Engelberg R, Martin DP, Agel J (1999) Short musculoskeletal function assessment questionnaire: validity, reliability, and responsiveness. J Bone Jnt Surg Am 81:12451260

16. Kellgren JH, Lawrence JS (1957) Radiological assessment of osteo-arthrosis. Ann Rheum Dis 16:494-502

17. Ahlback S (1968) Osteoarthrosis of the knee. A radiographic investigation. Acta Radiol Diagn (Stockh) Suppl-277:7-72

18. Karol LA, Halliday SE, Gourineni P (2000) Gait and function after intra-articular arthrodesis of the hip in adolescents. J Bone Jnt Surg Am 82:561-569

19. Joshi AB, Markovic L, Hardinge K, Murphy JC (2002) Conversion of a fused hip to total hip arthroplasty. J Bone Jnt Surg Am 84:1335-1341

20. Panagiotopoulos KP, Robbins GM, Masri BA, Duncan CP (2001) Conversion of hip arthrodesis to total hip arthroplasty. Instr Course Lect 50:297-305 